\title{
Product Landscape of Rapid Diagnostic Tests for Viral Hemorrhagic Fever Pathogens
}

\author{
Noah Hull², Andrew Hickey ${ }^{1}$ and Teresa Quitugua ${ }^{1}$ \\ 'National Biosurveillance Integration Center, Office of Health Affairs, United States Department of Homeland Security, Washington, \\ DC, USA; ${ }^{2}$ University of Wyoming, Laramie, WY, USA
}

\section{Objective}

Provide a succinct review of potential developmental and commercial viral hemorrhagic fever diagnostic assays published in peer-reviewed literature and open-source platforms.

\section{Introduction}

As the lead for coordinating domestic incident management across all Federal departments and agencies, Department of Homeland Security is responsible for identifying emergency response resources. The 2014 Ebola - Zaire outbreak in West Africa that became a Public Health Emergency of International Concern highlighted the need to understand the current and potential availability of diagnostic assays for a number of viral hemorrhagic fevers that may require similar response actions. A concatenated list of publically reported potential viral hemorrhagic fever diagnostic assays was therefore compiled. Etiologic agents in the families of Arena-, Bunya-, Filo-, and Flaviviridae were included in the analysis. This list identifies assays in various stages of development that could be submitted, at some point, to the Food and Drug Administration (FDA) under an Emergency Use Authorization (EUA) should such a need arise.

\section{Methods}

Boolean logic formatted searches were conducted using PubMed, Google Scholar, and Google to identify open-source reports of diagnostic assays for viral hemorrhagic fevers. The general Boolean search term was [(PATHOGEN OF INTEREST AND Diagnostic) (Rapid OR Point of Care OR “Assay Development" OR Validation OR POC OR Evaluation OR Clinical OR Deploy* OR Testing OR Detect*)]. Returned results were screened for data elements that provided adequate information to derive test performance statistics (limit of detection, sensitivity with $95 \%$ confidence interval, specificity with $95 \%$ confidence interval, positive predictive value, and negative predictive value). Assays were qualitatively analyzed based on limit of detection, sensitivity, and specificity estimates. Genus level etiologic agents by family are: Arenaviridae (Chapare, Guanarito, Junin, Lassa, Lujo, Lymphocytic choriomennigitis, Machupo, Sabia, and Whitewater Arroyo), Bunyaviridae (CrimeanCongo, Hantavirus, and Rift Valley Fever), Filoviridae (Ebola and Marburg), and Flaviviridae (Dengue, Kyasanur Forest Disease, Omsk, and Yellow Fever).

\section{Results}

Through the use of the Boolean logic, 312 unique assay data sources were identified and examined. One hundred seventeen sources contained adequate information to derive diagnostic test statistics for examination as part of this review. Inadequate information was identified for Chapare Hemorrhagic Fever, Whitewater Arroyo Virus, and Omsk Hemorrhagic Fever, so diagnostic information for these agents was not included in the final table. Assays were dichotomized as either academic/government or commercial assays. Of the 212 total assays, $136(64 \%)$ resided in academic or government laboratories. Of those, the vast majority addressed the Arena-, Bunya-, and Filoviridae families ( $35 \%, 34 \%$, and $26 \%$ respectively), compared to the commercial assays addressing the Filo- and Bunyaviridae families
(43\% and $27.6 \%$ respectively). The disproportionate weight of the commercial assays on Filoviridae is likely a function of the 2014 Ebola-Zaire outbreak. In the academic/government validated assays, $82 \%$ were validated on clinically derived samples, in comparison to $57 \%$ from the commercial assays. Clinically validated assays are the "gold-standard" validation technique as recommended by the FDA.

\section{Conclusions}

The potential product landscape for rapid diagnostics of viral hemorrhagic fevers is extensive. However, the vast majority of these assays were developed in academic or government laboratories and have not progressed further toward commercialization or broad public health use. Subsequently, should an EUA be sought for these diagnostic assays, their approval may be delayed due to the lack of validation and standardization required for deployment as a reliable diagnostic tool. Lastly, the validation methods by which each assay was tested varied greatly, resulting in difficultly drawing direct comparisons amongst assays.

\section{Keywords}

Viral Hemorrhagic Fever; Diagnostic Test; Product Landscape

\section{Acknowledgments}

This material is based upon work supported by the United States Department of Homeland Security - Office of Health Affairs - Volunteer Scholar Program and by the United States Department of Homeland Security under Cooperative Agreement Number DHS 2010-ST061-AG002.

\section{*Noah Hull}

E-mail: Nhull@uab.edu 